\title{
LOS PRIMEROS DESCUBRIMIENTOS DE DINOSAURIOS EN ESPAÑA
}

\author{
Xabier PEREDA SUBERBIOLA ${ }^{1}$ y José Ignacio \\ RUIZ-OMEÑACA
}

${ }^{1}$ Universidad del País Vasco/EHU, Facultad de Ciencia y Tecnología, Departamento de Estratigrafía y Paleontología, Apdo. 644, 48080 Bilbao.

xabier.pereda@lg.ehu.es

${ }^{2}$ Universidad de Zaragoza, Departamento de Ciencias de la Tierra, Área y Museo de Paleontología, 50009 Zaragoza. jigruiz@unizar.es

Pereda Suberbiola, X. \& Ruiz-Omeñaca, J. I. 2005. Los primeros descubrimientos de dinosaurios en España. [The first dinosaur discoveries in Spain.] Revista Española de Paleontología, N.E. X, 15-28. ISSN 0213-6937.

\begin{abstract}
The fi rst dinosaur discovery in Spain is a presumed theropod tooth from the Upper Jurassic of Asturias. It was described in 1858 as a shark tooth by the German mining engineer Guillermo Schulz and referred to the dinosaur Megalosaurus by the Navarrese geologist Justo Egozcue in 1873. However, the whereabouts of this fossil is currently unknown.

In 1872 and 1873, the Valencian naturalist Juan Vilanova was the fi rst to specifi cally mention the discovery of dinosaur fossil remains from Spain. He described Iguanodon bones from the Lower Cretaceous of Utrillas (Teruel) and Morella (Castellon). The Vilanova collection, currently kept in the Museo Nacional de Ciencias Naturales of Madrid, consists of fi ve fragmentary remains from Morella and, tentatively, one from Utrillas, but none is referable to Iguanodon. One of the Morella fossils is interpreted as a theropod vertebral centrum, a second one could represent the distal end of the neural spine of a diplodocoid sauropod, the other three remains are indeterminate bone fragments. The two "long bones" from Utrillas mentioned by Vilanova correspond to fragments of a theropod tibia, now lost, but recognizable from a drawing made around the 1920's by the Castellonese palaeontologist José Royo Gómez. The study of dinosaurs in Spain was not highlighted during the 1800's and the fi rst signifi cant discoveries were not made until the late 1910's.
\end{abstract}

Key words: Dinosauria, Vilanova, Schulz, Egozcue, XIX ${ }^{\text {th }}$ century, Spain.

\section{RESUMEN}

El hallazgo más antiguo conocido de un dinosaurio en España es un supuesto diente de terópodo del Jurásico Superior de Asturias. Fue descrito en 1858 como un diente de tiburón por el ingeniero de minas alemán Guillermo Schulz y asignado en 1873 al dinosaurio Megalosaurus por el geólogo navarro Justo Egozcue. Se desconoce el paradero actual de este fósil.

El naturalista valenciano Juan Vilanova publicó en 1872 y 1873 las primeras citas de dinosaurios en España. Vilanova mencionó el descubrimiento de restos fósiles del ornitópodo Iguanodon en el Cretácico Inferior de Utrillas (Teruel) y Morella (Castellón). La colección Vilanova depositada actualmente en el Museo Nacional de Ciencias Naturales de Madrid se compone de cinco especímenes fragmentarios de Morella y, con reservas, uno de Utrillas. Ninguna de las piezas puede atribuirse a Iguanodon. Uno de los fósiles de Morella se interpreta como un centro vertebral de un posible terópodo, y otro de ellos como la extremidad distal de una espina neural perteneciente a un saurópodo diplodocoideo; los otros tres fósiles son fragmentos de huesos indeterminados. Los dos «huesos largos» de Utrillas citados por Vilanova corresponden a dos fragmentos de una tibia de terópodo, actualmente desaparecida, de la que se conserva un dibujo realizado en torno a la década de 1920 por el paleontólogo castellonense José Royo Gómez. El estudio de los dinosaurios recibió poca atención en España durante el siglo XIX y los primeros descubrimientos relevantes no se realizaron hasta fi nales de la década de 1910.

Palabras clave: Dinosauria, Vilanova, Schulz, Egozcue, siglo XIX, España. 


\section{INTRODUCCIÓN}

No se sabe con certeza en qué fecha se descubrieron los primeros restos fósiles de dinosaurio. Se conocen referencias en las antiguas crónicas chinas del hallazgo de «huesos petrificados de dragón» que podrían haber sido en realidad restos fósiles de dinosaurios (Dong, 1988). Los textos griegos y romanos también contienen descripciones de hallazgos de fósiles de vertebrados, algunos de los cuales pudieron pertenecer a dinosaurios (Mayor, 2000). Además, hay constancia de que diferentes culturas humanas se han interesado por las evidencias fósiles de dinosaurios, ya que se conocen localidades con arte rupestre situadas cerca de yacimientos con icnitas de dinosaurios (Lockley, 1991; Sarjeant, 1997) y abalorios arqueológicos elaborados a partir de fragmentos de cáscara de huevo de dinosaurio (Andrews, 1932).

La ilustración más antigua conocida de un hueso fósil de dinosaurio data del siglo XVII (Plot, 1677). Se trata de un fémur de terópodo descubierto en el Jurásico de Inglaterra, aunque fue identificado en aquella época como un «hueso petrificado» de elefante (Sarjeant, 1997) o perteneciente a un gigante (Halstead \& Sarjeant, 1993). Este mismo fósil, hoy perdido, fue descrito posteriormente con el nombre de Scrotum humanum por Brookes (1763). Basándose asimismo en fósiles del Jurásico inglés, Lhuyd (1699) ilustró un diente aislado de terópodo y otro de saurópodo, interpretándolos como «imitaciones de dientes de peces» (Delair \& Sarjeant, 2002). Otros hallazgos esporádicos tuvieron lugar en la Inglaterra del siglo XVIII (Delair \& Sarjeant, 1975, 2002; Sarjeant, 1997). No obstante, el estudio de los dinosaurios no alcanzó un verdadero impulso en Europa hasta principios del siglo XIX (Colbert, 1968; Cadbury, 2000). Durante la década de 1820 se publicaron en Inglaterra las primeras descripciones científicas: William Buckland (1824) erigió Megalosaurus y Gideon A. Mantell (1825) formalizó Iguanodon. Cuando Richard Owen acuñó el término Dinosauria en 1842, se habían descrito nuevos géneros (Hylaeosaurus, Plateosaurus, Poikilopleuron, Thecodontosaurus y Cetiosaurus, entre otros -aunque sólo Megalosaurus, Iguanodon e Hylaeosaurus fueron reconocidos por Owen como dinosaurios-) y el descubrimiento de restos fósiles de dinosaurios había alcanzado Alemania en 1832 (Probst \& Windolf, 1993) y Francia en 1838 (Buffetaut et al., 1993; Allain \& Chure, 2002). Previamente, Georges Cuvier $(1808,1824)$ había descrito y figurado varias vértebras de un dinosaurio terópodo descubiertas en la década de 1770 en el Jurásico de Normandía como pertenecientes a un cocodrilo marino (Taquet, 1984; Allain, 2001). En Rusia, los primeros restos fósiles de dinosaurios se publicaron en 1834, pero la descripción sistemática de nuevo material no tuvo lugar hasta el siglo XX (Chure \& McIntosh, 1989). Durante las décadas de 1850 y 1860 , se anunció el hallazgo de restos fósiles de dinosaurio en otros países europeos como Suiza y Austria, así como en los Estados Unidos, Sudáfrica, Brasil y la India (Chure \& McIntosh, 1989). El registro fósil norteamericano había proporcionado evidencias fósiles (huesos y huellas) de dinosaurio con anterioridad, pero estos descubrimientos no fueron reconocidos hasta mucho más tarde (Colbert, 1968; Lockley, 1991). Por lo que respecta a los huevos de dinosaurio, las primeras cáscaras fósiles descritas en la literatura fueron halladas en Provenza en 1846 (Taquet, 2001) y, poco después, en 1859, en los Pirineos gascones (Buffetaut \& Le Loeuff, 1994), pero estos hallazgos pasaron desapercibidos hasta el descubrimiento de nidos de dinosaurio en Mongolia durante las expediciones realizadas por el American Museum of Natural History de Nueva York en la década de 1920 (Andrews, 1932).

En la Península Ibérica, la ilustración más antigua que se conoce relacionada con dinosaurios es un embaldosado del siglo XVIII en el que aparecen representadas huellas de saurópodo de un acantilado de Cabo Espichel en Portugal (Antunes \& Mateus, 2003). La primera mención de fósiles de dinosaurio en España tuvo lugar a principios de la década de 1870 (Vilanova, 1872, 1873; Egozcue, 1873). El relato de estos hallazgos ha sido tratado brevemente por autores modernos (Santafé et al., 1982; Sanz, 1984, 1996; Sanz et al., 1990; Santafé \& Casanovas, 1993; Casanovas, 1993; Alcalá \& Alcalá, 1996; Gasulla, 2002; Ruiz-Omeñaca et al., 2003). En este trabajo se discuten aspectos relacionados con la primera cita y el hallazgo más antiguo de fósiles de huesos de dinosaurio en España de acuerdo con los datos actualmente disponibles. Asimismo, se revisa por primera vez la colección Vilanova de dinosaurios depositada en el Museo Nacional de Ciencias Naturales (MNCN-CSIC) de Madrid.

\section{LA PRIMERA CITA DE UN DINOSAURIO EN ESPAÑA}

La cita más antigua de un dinosaurio en España de la que se conoce documentación escrita data de 1872. Ese año se publicó el Compendio de Geología (Fig. 1), obra del naturalista valenciano Juan Vilanova Piera (Fig. 2). En la página 477 del mismo puede leerse (Vilanova, 1872; citado por Alcalá \& Alcalá, 1996):

"Para concluir, debo mencionar el hallazgo hecho tres años há por D. Nicolás Ferrer, y confirmado más tarde por mí, de varios restos de un reptil colosal al pié de la colina llamada Benigania, junto á los muros mismos de Morella. Tambien poseo dos huesos largos, que probablemente pertenecen al Ignanodon [sic] Mantelli que, procedentes de Utrillas, me mandó hace poco el distinguido médico de Montalban D. Jerónimo Balduque. Quizás sean estos los únicos huesos de reptiles cretáceos hasta el presente encontrados en la Península, razon que me ha movido á dar estas noticias." 


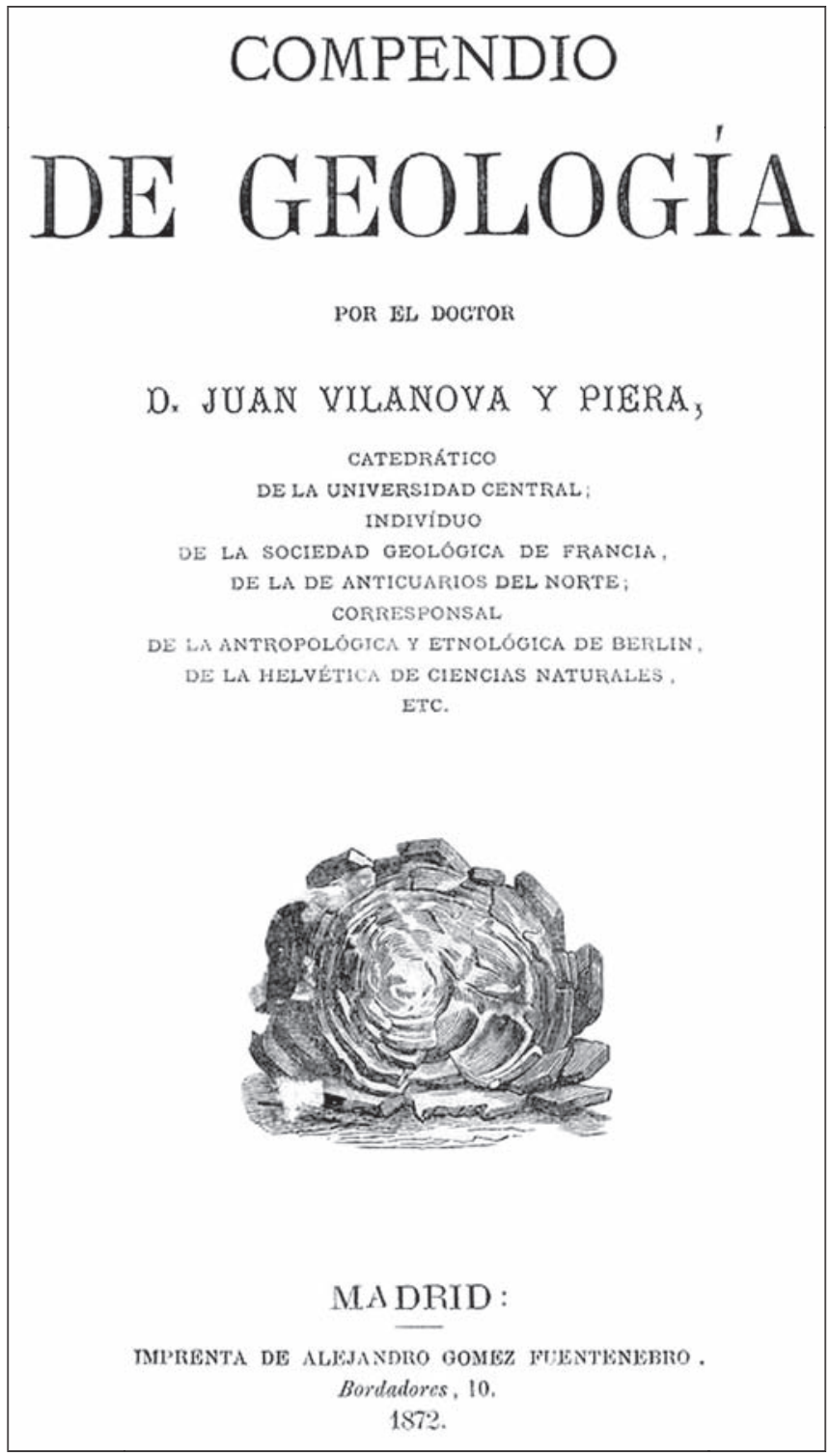

Figura 1. Portada del Compendio de Geología de Vilanova (1872). Incluye la cita más antigua de un dinosaurio en España de la que se conoce documentación escrita (p. 477). Title page of the Compendio de Geología by Vilanova (1872). This book ( $p$. 477) contains the earliest dinosaur mention in Spain based on written documents.

Este mismo texto fue reproducido por el propio Vilanova en la página 290 del tomo VIII de La Creación, publicado en 1876 (R. Gozalo, com. pers.). Curiosamente, cuando en las páginas 432 a 434 del mismo tomo describe el Orden Dinosáuridos, no hace referencia a los hallazgos en España (Vilanova, 1876).

Vilanova comunicó a la Sociedad Española de Paleontología, durante la sesión del 5 de febrero de 1873, celebrada en Madrid bajo la presidencia del Sr. Pérez Arcas, el descubrimiento de restos de dinosaurio en Utrillas (Teruel) y Morella (Castellón). La cita completa es la siguiente (Vilanova, 1873):

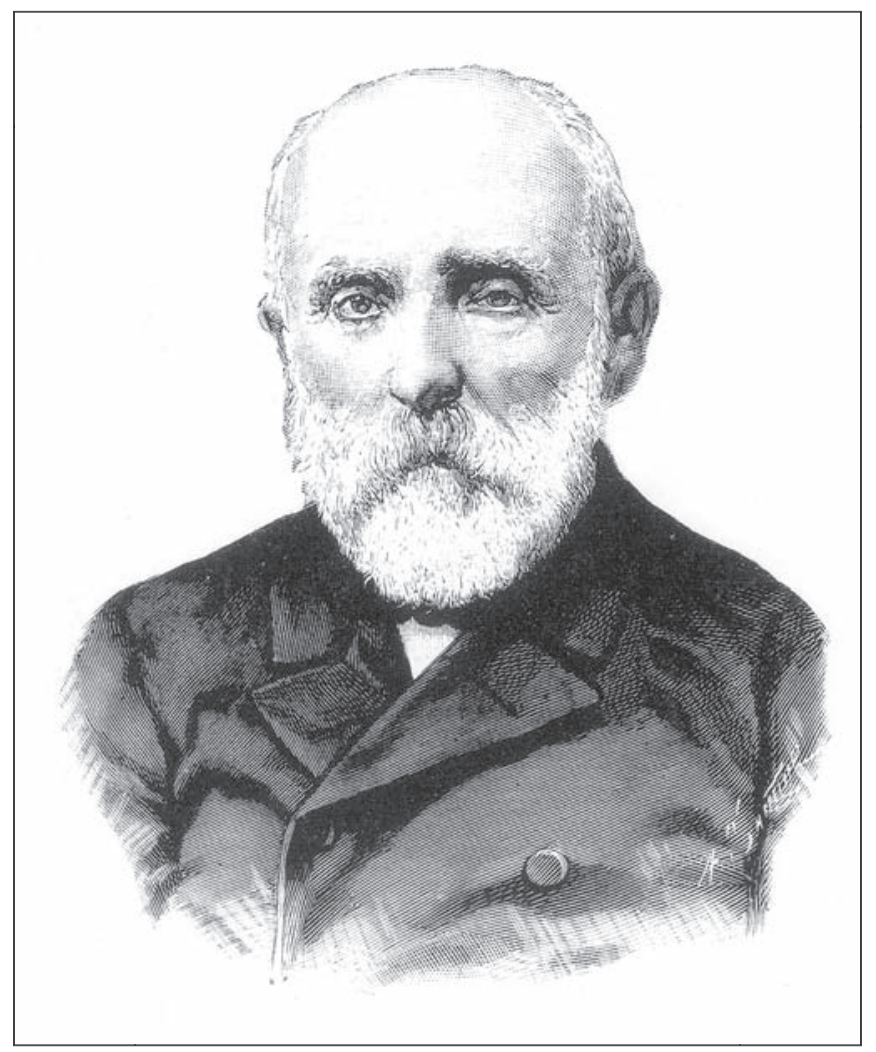

Figura 2. Retrato de Juan Vilanova Piera (Valencia, 5-V-1821; Madrid, 7-VI-1893). Tomado de Quiroga (1893). Portrait of Juan Vilanova Piera (Valencia, 5-V-1821; Madrid, 7-VI-1893). After Quiroga (1893).

"El señor Vilanova (D. Juan) participa á la Sociedad hallarse hace algún tiempo en posesión de los únicos restos fósiles, hasta ahora conocidos en España, segun cree, de Iguanodon, y son dos huesos largos de las extremidades anteriores, procedentes del lignito de Utrilla, y otro hueso tambien largo, de Morella."

De estas reseñas se desprende que el material de Morella fue descubierto a finales de la década de 1860 y consistía en un número indeterminado de especímenes (al menos un hueso largo). El historiador Francisco Ortí Miralles (1958: 20) señala que el Catedrático de Valencia D. Nicolás Ferrer Julve halló «restos de un esqueleto de dinosaurio» en Morella en 1868 (citado por Santafé et al., 1982). Esta información fue extraída de una obra de José Segura Barreda, publicada en 1868, en la que se dice que Ferrer Julve «encontró en agosto de 1868 algunos huesos disformes en las cercanías de Morella, que conservaban no sólo la figura exterior, sino hasta la médula fosilizada» (Segura Barreda, 1868: 280; citado por Santafé et al., 1982). Además del material de Morella, Vilanova $(1872,1873)$ identificó dos huesos largos de las extremidades anteriores procedentes de Utrillas y los asignó al ornitópodo Iguanodon. Poco después, José J. Landerer, científico aficionado de Valencia y, probablemente, alumno de geología de campo de Vilanova 
(Gozalo \& Navarro, 1995), menciona el descubrimiento de los restos de Iguanodon en Utrillas (Landerer, 1874: 364). En este mismo trabajo, Landerer (1874, dentro del epígrafe «Catálogo de las especies del piso tenéncico», p. 375) cataloga el Iguanodon de Vilanova como perteneciente a una nueva especie. Poco tiempo después, Calderón (1876, 1877) menciona el descubrimiento de Iguanodon hecho por Vilanova en Utrillas (Teruel). Curiosamente, Iguanodon no es mencionado por Mallada $(1887,1892,1904)$ en sus catálogos sobre las especies fósiles de España (de hecho, sólo menciona entre los vertebrados mesozoicos tres peces: Sphenodus, Pycnodus y Lamna).

Los restos de dinosaurios de Morella y Utrillas citados por Vilanova (o, al menos, parte de la colección) se depositaron en el Museo Nacional de Ciencias Naturales (MCNA-CSIC) de Madrid, según consta en las publicaciones del paleontólogo castellonense José Royo Gómez (Alcalá \& Alcalá, 1996; Alcalá, 2004). La última referencia conocida acerca de su localización en la «sala del Diplodocus» aparece en los trabajos de Royo Gómez con motivo del Congreso Geológico Internacional celebrado en Madrid en 1926 (Royo Gómez, 1926, 1928; Alcalá, 2004 y com. pers.) A este respecto, Montero (2003: 364) indica la existencia de un inventario manuscrito e incompleto de la «sala del Diplodocus» en los años 1920.

\section{Sobre los «dos huesos largos» de Utrillas (Teruel)}

Vilanova $(1872,1873)$ únicamente mencionó como procedentes de Utrillas «dos huesos largos», aunque otros autores mencionan dientes (Bataller, 1960: 161; Sanz, 1998: 17), confundiendo probablemente la cita de Vilanova con otras posteriores de Royo Gómez de restos fósiles de Morella.

Estos «dos huesos largos» han sido citados de distinta manera y se han asignado a diferentes taxones. Royo Gómez (1920: 266; 1921: 227) los mencionó manteniendo su asignación a Iguanodon. Posteriormente, el propio Royo Gómez $(1926,1928)$ [el trabajo de 1926 es una traducción al castellano del trabajo de 1928, que aunque fue redactado antes se publicó más tarde, con pequeñas diferencias] los citó como «dos fragmentos de un fémur de un dinosaurio pequeño, que por error [Vilanova] determinó como de Iguanodon» (Royo Gómez, 1926: 173; véase también Royo Gómez, 1928: 2040). Sin descartar que pertenecieran a un ornitópodo, Royo Gómez (1926: 176; 1928: 2042) los asignó «a una forma pequeña y quizás nueva» (Royo Gómez, 1926: 176; 1928: 2042). Por último, Royo Gómez (1927: 127) atribuyó los fósiles a un terópodo:

"J'ai encore quelques pièces de différents gisements parmi lesquelles je citerai un tibia de Théropode d'Utrillas (prov. de Teruel) ayant à peu près $25 \mathrm{~cm}$. de longueur, qui peut montrer l'existence à ce temps-là d'une forme à taille très petite".
Por su parte, Bataller (1960) los asignó a Iguanodon sp., aunque también mencionó Trachodon [un hadrosaurio] en Utrillas, citando a Royo Gómez (1928).

Las interpretaciones de Royo Gómez han sido citadas de manera incorrecta en varios trabajos. Así, Lapparent (1960: 17) dijo que «... en Utrillas, en los lignitos del Albiense y no del Wealdiense, Vilanova (1873) dio cuenta de una tibia de Terópodo carnívoro» [refiriéndose por error a Vilanova (1873) en lugar de a Royo Gómez (1927), siendo ambos trabajos citados en la bibliografía de Lapparent], mientras que Bataller (1960: 161) concluyó que «Iguanodon sp. (...) fue la primera forma de dinosaurio reconocida en España (...) el de Utrillas por Vilanova (1873), consistente en un femur de dinosaurio pequeño que atribuye a este género», haciendo referencia a Royo Gómez (1928).

Aunque Royo Gómez (1927) no lo dice explícitamente, se puede asumir que la tibia de terópodo de Utrillas es el mismo fósil que los «dos huesos largos» de Vilanova $(1872,1873)$ y que, por lo tanto, estos últimos eran en realidad los extremos proximal y distal de dicha tibia. Este resto nunca ha sido descrito, ni figurado, aunque se conserva un dibujo del mismo (Fig. 3) en un manuscrito inédito de Royo Gómez (Ruiz-Omeñaca \& Pereda Suberbiola, 1999). Dado que la tibia se halló en los lignitos de Utrillas (Vilanova, 1873), hoy incluidos en la Formación Escucha, su edad sería Albiense inferior-medio (Querol et al., 1992).

La tibia de Utrillas no ha podido ser localizada en los diferentes museos de Madrid y Valencia en los que pudo haber sido depositada (Museo Nacional de Ciencias Naturales de Madrid, Museo de Geología de la Universidad de Valencia, Museo Municipal de Ciencias Naturales de Valencia). Si el material estaba en Valencia, pudo destruirse en el incendio del antiguo Museo de Historia Natural de la Universidad de Valencia (A. García Forner y M. Belinchón, com. pers.), sucedido el 12 de mayo de 1932 (Navarro \& Catalá, 2000; García Forner et al., 2002).

\section{La colección Vilanova de dinosaurios del Museo Nacional de Ciencias Naturales de Madrid}

La colección Vilanova de dinosaurios consiste en cinco restos fósiles sin fecha de depósito, catalogados con los números de inventario MNCN 609, 610, 611, 612 y 31051. Según la base de datos del MNCN, todos estos restos proceden del Cretácico Inferior de Morella. Una de las piezas (MNCN 610) conserva una etiqueta antigua, escrita a mano por Royo Gómez, en la que se lee «(Facies Weáldica), Morella (Castellón), Col. Vilanova (Huesos de Iguanodon)» (Fig. 4). Los restantes especímenes presentan etiquetas modernas con la mención «frag. hueso dino- 


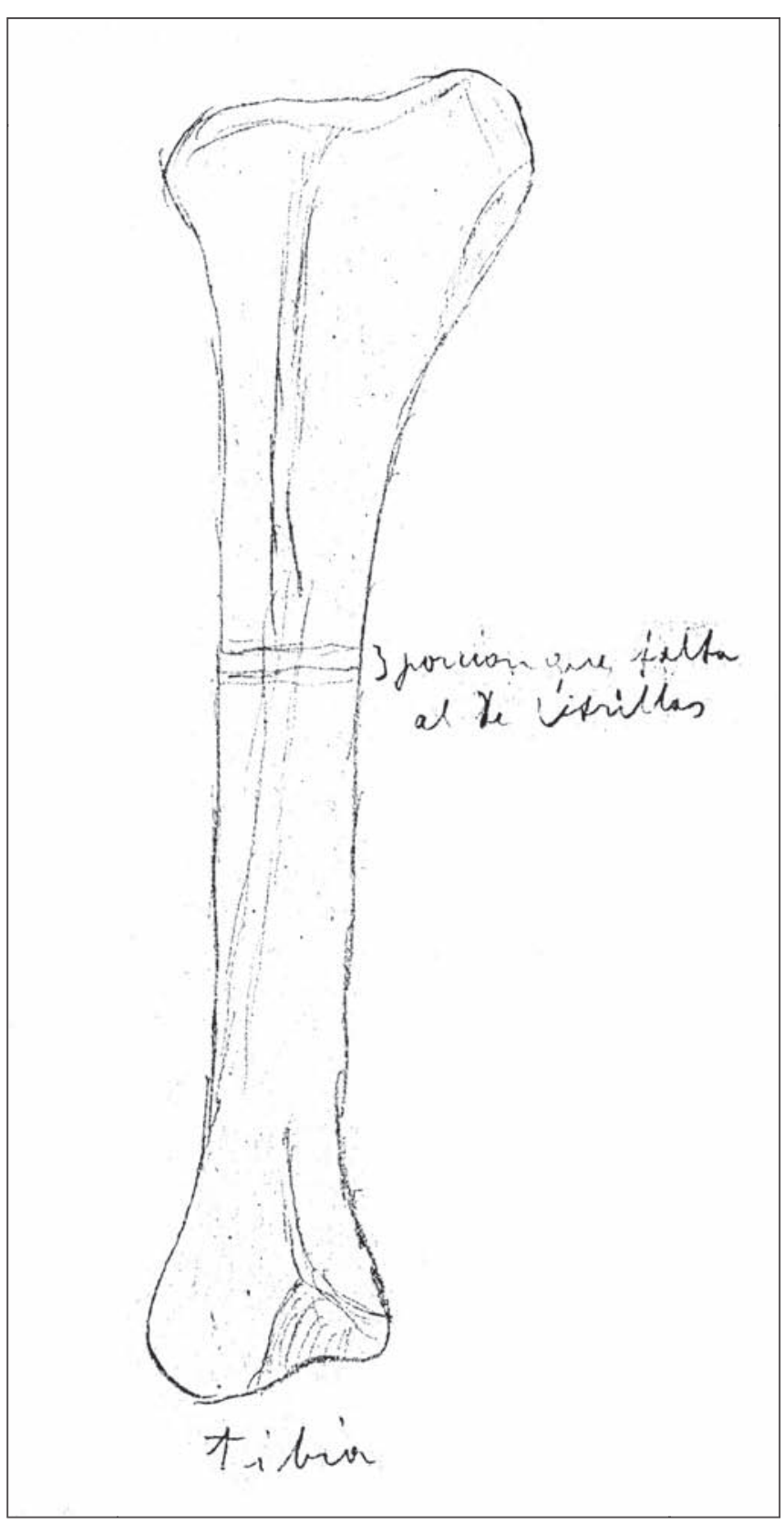

Figura 3. Dibujo de Royo Gómez de la tibia de terópodo de Utrillas, mencionada como Iguanodon por Vilanova. Colección Royo Gómez, documento c.4-P137, “J. Royo y Gómez. Trabajos y apuntes científicos, /sin fecha/". Cortesía del Archivo del Museo Nacional de Ciencias Naturales de Madrid.

The theropod tibia from Utrillas cited as Iguanodon by Vilanova. Drawing by José Royo Gómez. Royo Gómez collection, document c.4-P137, "J. Royo y Gómez. Trabajos y apuntes científicos,/sin fecha/". Reproduced with permission of the Archivo del $\mathrm{Mu}$ seo Nacional de Ciencias Naturales, Madrid.

saurio, Col. Vilanova, Morella», excepto la pieza MNCN 31052, cuya etiqueta reza: «Reptil sp., Frag. hueso, Morella (Castellón), Weáldico».

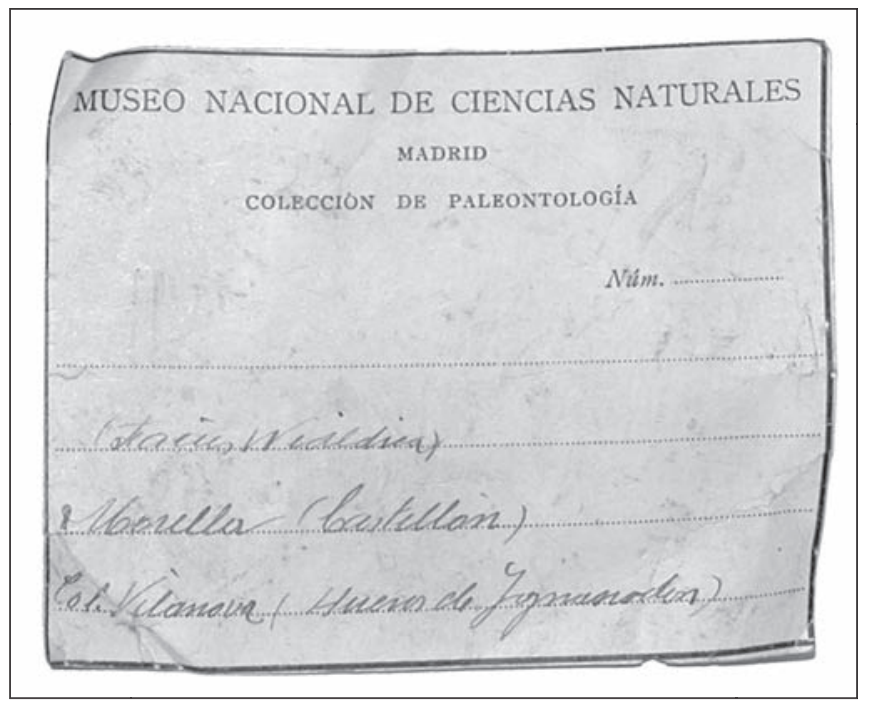

Figura 4. Etiqueta antigua del Museo Nacional de Ciencias Naturales correspondiente a la pieza MNCN 610, escrita a mano por José Royo Gómez. Cortesía del Museo Nacional de Ciencias Naturales de Madrid. Old label for the specimen MNCN 610, handwritten by José Royo Gómez. Reproduced with permission of the Museo Nacional de Ciencias Naturales, Madrid.

Los fósiles son de color grisáceo rojizo, con excepción de MNCN 31051 que presenta un color más claro, con una pátina ocre. Algunos de ellos conservan una matriz verdosa de carácter areniscoso en algunas áreas de su superficie. Se trata de piezas fragmentarias, cuya dimensión máxima no supera en ningún caso los $15 \mathrm{~cm}$ y de difícil reconocimiento. Se han identificado dos restos vertebrales, que consisten en un centro y un fragmento de espina neural. Tres restos son indeterminados, aunque dos de ellos podrían corresponder a costillas. Una de las piezas (MNCN 610) podría ser el «hueso largo» mencionado por Vilanova (1873).

Se desconoce el lugar exacto de recogida de los fósiles, pero su color rojizo sugiere que provienen de las denominadas Capas rojas de Morella. Este término se utiliza para referirse de manera informal a la Formación Arcillas de Morella, una intercalación continental dentro de una serie marina esencialmente carbonatada, situada estratigráficamente por encima de las facies Weald s. s. (Canerot et al., 1982). De coloración rojiza, las facies de esta formación se interpretan como un sistema deposicional de llanura fangosa (mud-flat o barrizal) y fluvial con escasa influencia mareal (Gàmez et al., 2003). Se trata de un episodio sedimentario regresivo en la base del Aptiense inferior (Santafé et al., 1982).

Aunque Vilanova no hizo mención de restos vertebrales, el espécimen MNCN 611 (Fig. 5a-d) se identifica como un fragmento de centro vertebral (longitud conser- 
vada: $60 \mathrm{~mm})$. Conserva una de las caras articulares, sin que pueda decirse si se trata de la anterior o posterior (diámetro máximo: $95 \mathrm{~mm}$ ). La superficie articular es plana a ligeramente cóncava (Fig. 5a) y está muy expandida con respecto al resto del centro. Los bordes están bien definidos y presentan rugosidades en forma de pequeñas crestas incipientes, sobre todo en la región ventral (Fig. 5d). No hay evidencia de pleurocelos. El centro está muy comprimido lateralmente (diámetro horizontal: $50 \mathrm{~mm}$ ) y tiene forma de carrete en vista ventral. El fósil está seccionado y pulido en su parte media (Fig. 5b) y muestra una superficie interna neumatizada (Fig. 5c). La región ventral es muy estrecha y no posee quilla. En vista dorsal, se observa una superficie erosionada en la zona de sutura para el arco neural. La ausencia de parapófisis, quilla ventral y superficies de articulación para los arcos hemales sugiere que la vértebra procede de la región dorsal media o posterior. La forma general de la vértebra, con un centro muy comprimido lateralmente y un perfil ventral fuertemente cóncavo en vista lateral, sumado al hecho de estar neumatizada internamente sugieren que la vértebra perteneció a un dinosaurio terópodo (Madsen, 1976; Holtz, 2000). No obstante, la pieza es muy fragmentaria para una determinación precisa. Las dimensiones del espécimen son equivalentes a las de la séptima vértebra dorsal de Allosaurus (Madsen, 1976).

Por otro lado, MNCN 610 (Fig. 5g-j) es una pieza mazuda que se interpreta como la extremidad distal de la espina neural de una vértebra (altura conservada: $140 \mathrm{~mm}$ ). La neurapófisis es más ancha transversalmente $(85 \mathrm{~mm})$ que en sentido anteroposterior $(60 \mathrm{~mm})$ (Fig. 5hi), una característica de las vértebras dorsales de la mayoría de los saurópodos (Wilson, 2002). Como es típico en este grupo de dinosaurios, se observa la presencia de una lámina preespinal (Fig. 5g). Además, hay una lámina lateral definiendo una pequeña cavidad situada por debajo del borde superior. No hay evidencia de lámina postespinal pero, en esta zona, el hueso está erosionado (Fig. 5j). En sección, la neurapófisis muestra dos cavidades internas de forma irregular (Fig. 5i). Su forma de pétalo en vista an- terior o posterior (Fig. $5 \mathrm{~g}, 5 \mathrm{j}$ ), con bordes más estrechos hacia la base que hacia la extremidad distal, es frecuente en las vértebras dorsales y caudales anteriores de diplodocoideos como rebaquisáuridos y dicraeosáuridos (Calvo \& Salgado, 1996; Upchurch, 1998; Wilson, 2002). A pesar del carácter fragmentario de la pieza, ésta se asigna provisionalmente a un saurópodo diplodocoideo.

Las otras piezas de Morella pertenecientes a la colección Vilanova corresponden a restos fósiles indeterminados. MNCN 609 (Fig. 5k) (dimensiones en sección transversal: 90 y $58 \mathrm{~mm}$ ) y MNCN 612 (Fig. 5l) (longitud: $140 \mathrm{~mm}$; anchura: $85 \mathrm{~mm}$ ) podrían representar fragmentos de costillas. MNCN 31051 (Fig 5e) es un fragmento de hueso indeterminado (longitud: $90 \mathrm{~mm}$; anchura $50 \mathrm{~mm}$ ). Se pone en duda la atribución de cualquiera de los restos de la colección Vilanova a Iguanodon, lo que no es óbice para destacar la presencia de este taxón en varias localidades de la región de Morella (Santafé et al., 1982; RuizOmeñaca \& Santos Cubedo, 1998; Gasulla, 2002).

En el MNCN de Madrid, se conserva un resto fósil procedente de Utrillas (Teruel), catalogado con el número de inventario MNCN 32653, que podría pertenecer a la colección Vilanova (Alcalá, 1998, 2002). Se ignora el año de recogida o de depósito. Se trata de un resto de forma plana (dimensiones conservadas medidas perpendicularmente entre sí: 140, 125 y 45 mm) de difícil identificación (Fig. 5f). Aunque no se descarta que se trate de un fragmento de diáfisis de un hueso del esqueleto apendicular de un dinosaurio, el resto es muy fragmentario para una atribución fiable.

\section{EL HALLAZGO MÁS ANTIGUO DE UN DINOSAURIO EN ESPAÑA}

En la década de 1870, el anuncio del descubrimiento de restos fósiles en el Maestrazgo generó nuevas citas de dinosaurios ibéricos. En la sesión del 4 de Junio de 1873 de la Sociedad Española de Paleontología, celebrada en Madrid, el naturalista navarro Justo Egozcue Cía dio cuenta

Figura 5. Especímenes de dinosaurios conservados en la colección Vilanova del Museo Nacional de Ciencias Naturales (MNCNCSIC) de Madrid. a-d: MNCN 611, Theropoda indet., centro vertebral fragmentario en vistas anterior o posterior (a), lateral (b) y ventral (d) y sección transversal (c); e: MNCN 31051, hueso indeterminado; f: MNCN 32653, hueso indeterminado (¿fragmento de diafisis?); g-j: MNCN 610, Diplodocoidea indet., extremidad distal de espina neural en vistas anterior (g), dorsal (h), ventral (i) y posterior (j); k-1: MNCN 609 y 612, huesos indeterminados (ifragmentos de costillas?) en vista dorsal (?). a-e y g-l provienen de Morella (Castellón) y f de Utrillas (Teruel). Escala gráfica: 50 mm. Cortesía del Museo Nacional de Ciencias Naturales de Madrid.

Dinosaur specimens from the Vilanova colection, kept at the Museo Nacional de Ciencias Naturales, Madrid (MNCNCSIC). a-d: MNCN 611, Theropoda indet., fragmentary centrum in anterior or posterior (a), lateral (b) and ventral (d) views; the same specimen in cross-section (c); e: MNCN 31051, indeterminate bone; $f$ : MNCN 32653, indeterminate bone (shaft fragment?); $g-j:$ MNCN 610, Diplodocoidea indet., distal end of a neural spine in anterior $(g)$, dorsal (h), ventral (i) and posterior (j) views; $k$-l: MNCN 609 y 612, indeterminate bones (rib fragments?) in dorsal view (?). a-e and g-l come from Morella (Castellón) and from Utrillas (Teruel). Scale bar: $50 \mathrm{~mm}$. Reproduced with permission of the Museo Nacional de Ciencias Naturales, Madrid. 

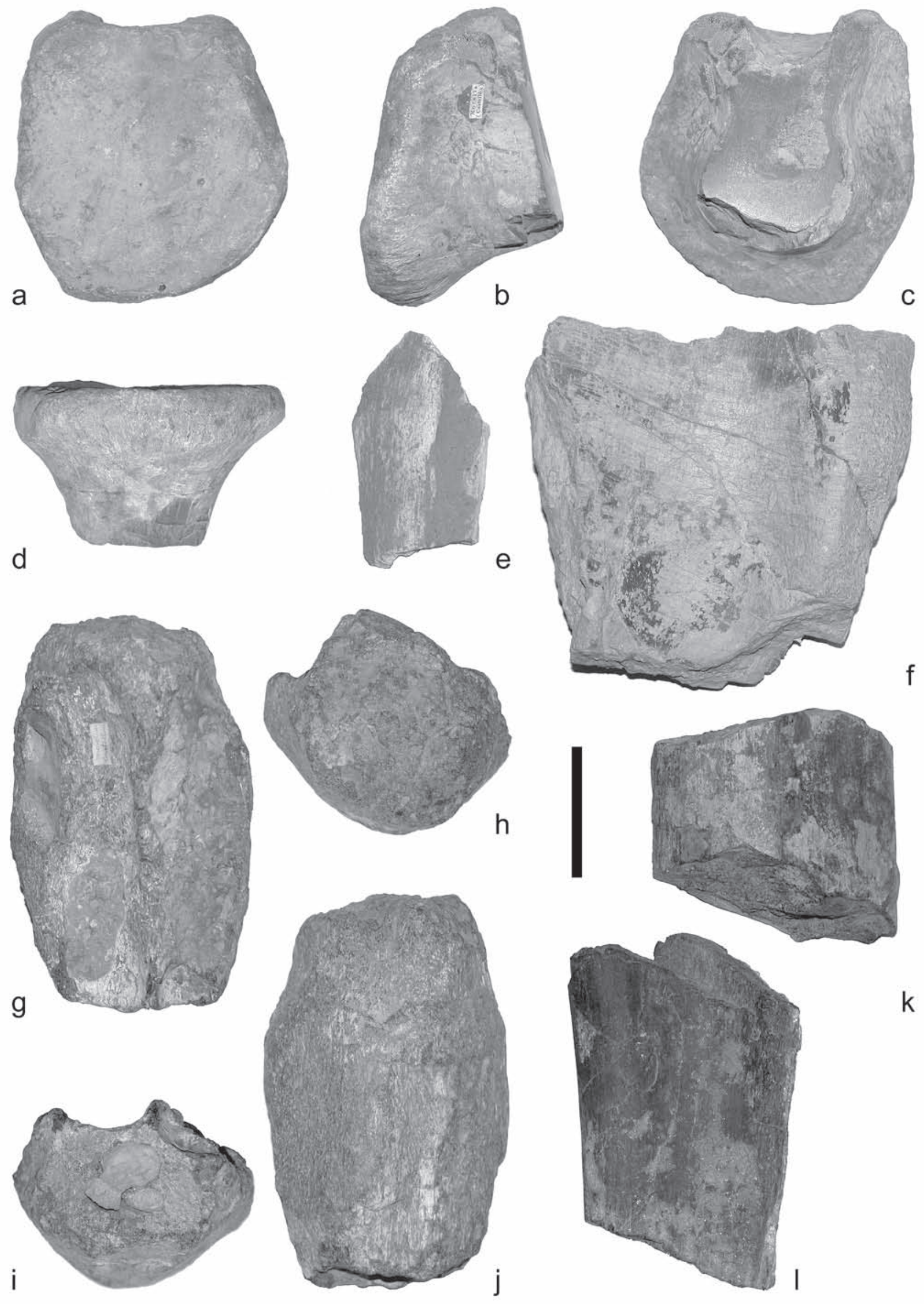

k 
de otro descubrimiento, anterior al de Vilanova, procedente de Asturias. La cita completa se retranscribe a continuación (Egozcue, 1873):

"El señor Egozcue dice, que con motivo de haber leido con sumo interés en el acta de la sesión del 5 de Febrero último que el Sr. Juan Vilanova posee unos huesos largos de Iguanodon, hallados en la zona de los lignitos cretáceos de Utrillas, le habia ocurrido presentar á la Sociedad, como lo hace, un diente de Megalosaurus, que en las colecciones de la Escuela de Minas venia figurando como correspondiendo á un animal de una clase muy distinta, y que, procedente tambien de una localidad española, creia se examinaria con gusto, en atencion á la suma rareza de ejemplares de restos fósiles de reptiles citados hasta ahora en nuestro suelo, y á ser esta la primera vez que públicamente se menciona en él la pasada existencia del género á que indudablemente corresponde. Agrega que ese diente tiene un doble interés si se considera la antigüedad del yacimiento en que se recogió. No es en efecto, añade, el ejemplar á la vista de los señores socios, sino el que, atribuido á un Squalus en la pág. 109 de la interesantísima DESCRIPCION GEOLOGICA DE ASTURIAS por el Sr. Schulz, se halló por el Sr. D. José de Elduayen en las margas rubias del grupo liásico, en término de Ruedes, á dos leguas cortas al Sur de Gijón; y como por los demas fósiles que el autor del susodicho trabajo menciona recogidos en las mismas margas hay que deducir que cuando menos corresponden al tramo medio del grupo liásico (ó sea al verdadero liásico de d'Orbigny), resulta que esa es tambien por lo menos la edad geológica del Megalosaurus á que perteneció el repetido diente; circunstancia que en la época en que se halló (ántes del año 1858) no hubiera dejado de llamar la atención, pero que ya hoy no es tan sorprendente, toda vez que M. J. Martin cita ese género (Bulletin de la Soc. géol. de France, t. XXII, p. 385), á un nivel todavía algo más bajo: al de la Aricula [sic] Contorta."

El geólogo de origen alemán Guillermo Schulz (Fig. 6) fue una de las figuras más sobresalientes de la geología asturiana del siglo XIX. Fue director de la Escuela de Minas de Madrid y de la Comisión del Mapa Geológico de España entre 1853 y 1857 (López de Azcona, 1984; Truyols, 1995). La descripción hecha por Schulz (1858) en la página 109 de su Descripcion geológica de Asturias (Fig. 7) del supuesto diente de dinosaurio al que alude Egozcue es la siguiente:

"Al terminar aquí por ahora nuestras indicaciones sobre los fósiles del Lías de Asturias, debemos hacer mención de un admirable diente de Squalus (primitivo tiburon) hallado por el $\mathrm{Sr}$. D. José de Elduayen en las margas rubias del Lías en términos de Ruedes á dos leguas cortas Sud de Gijon, este diente, cuya base y punta faltan, tendría (cuando completo) por lo menos cuatro pulgadas de largo, es de color pardo obscuro, por fuera muy reluciente y está adornado en ambos filos de finísima serreta; pero la del filo de curva convexa es algo más obtusa y no llega hasta la base, pues termina primorosamente á unos cuatro centímetros de ella."

Siguiendo la opinión de Egozcue, Calderón (1876, 1877) y Mallada (1902), discípulo predilecto de Egozcue,

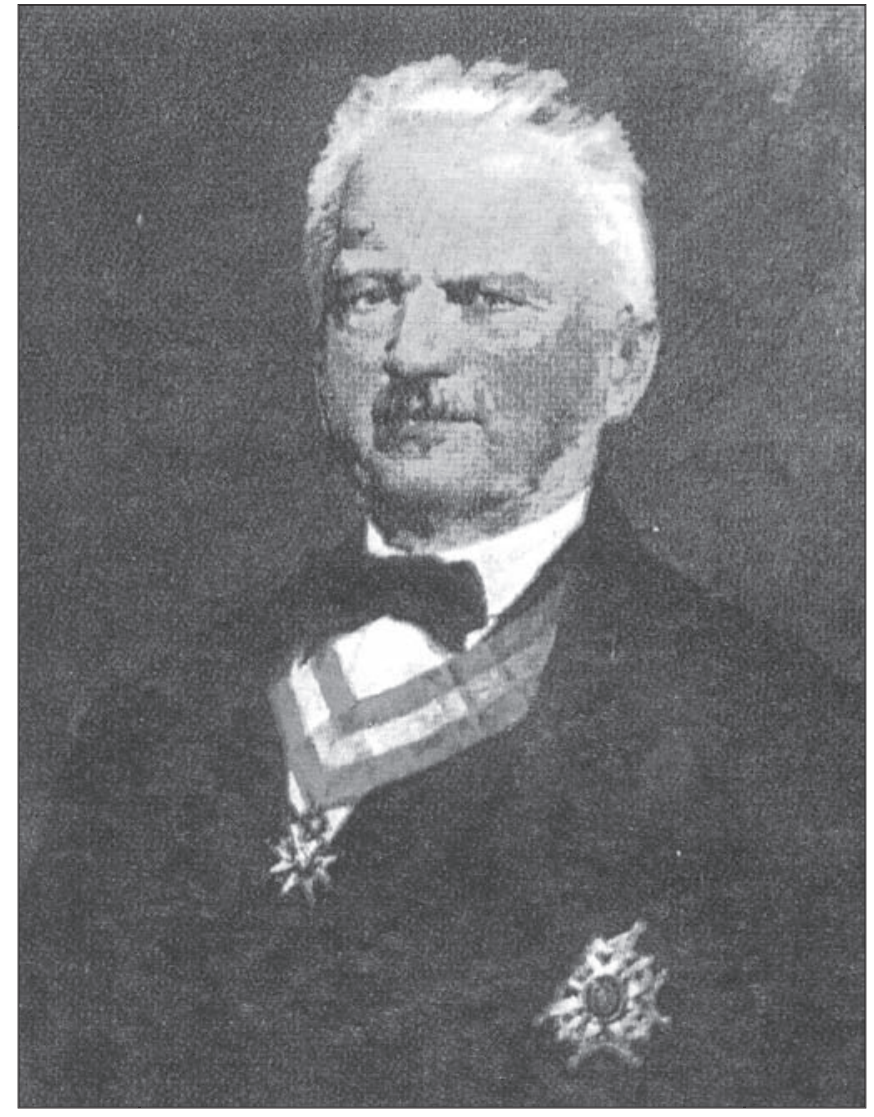

Figura 6. Retrato de Guillermo Schulz Schweizer (Habichtswald, 13-VI-1800; Aranjuez, 1-VIII-1877). Tomado de Puche Riart \& Ayala-Carcedo (2001). Cuadro original en la Escuela Técnica Superior de Ingenieros de Minas de Madrid.

Portrait of Guillermo Schulz Schweizer (Habichtswald, 13-VI-1800; Aranjuez, 1-VIII-1877). After Puche Riart \& Ayala-Carcedo (2001). Original painting in the Escuela Técnica Superior de Ingenieros de Minas, Madrid.

también citaron el diente de Asturias como perteneciente a Megalosaurus o a un megalosauro [sic], respectivamente, aunque, en un trabajo anterior, Mallada (1885) no lo citó.

La forma curvada del diente y la presencia de bordes crenulados sugieren que se trata probablemente de un dinosaurio carnívoro (aunque estos caracteres no son exclusivos de los terópodos; Currie, 1997). Su tamaño, con una corona de unos $10 \mathrm{~cm}$ de longitud, indica un animal de grandes dimensiones. No obstante, no existe ningún dibujo de la pieza, por lo que la interpretación de Egozcue, aunque es posible, no puede confirmarse. En varias ocasiones se ha citado la presencia de Megalosaurus en la Península Ibérica (véase Sanz, 1984 y referencias), pero está basada en restos aislados y poco diagnósticos, por lo que debe ser puesta en duda hasta que no se descubra material más completo. Megalosaurus fue el primer dinosaurio descrito y es uno de los más citados en la literatura 


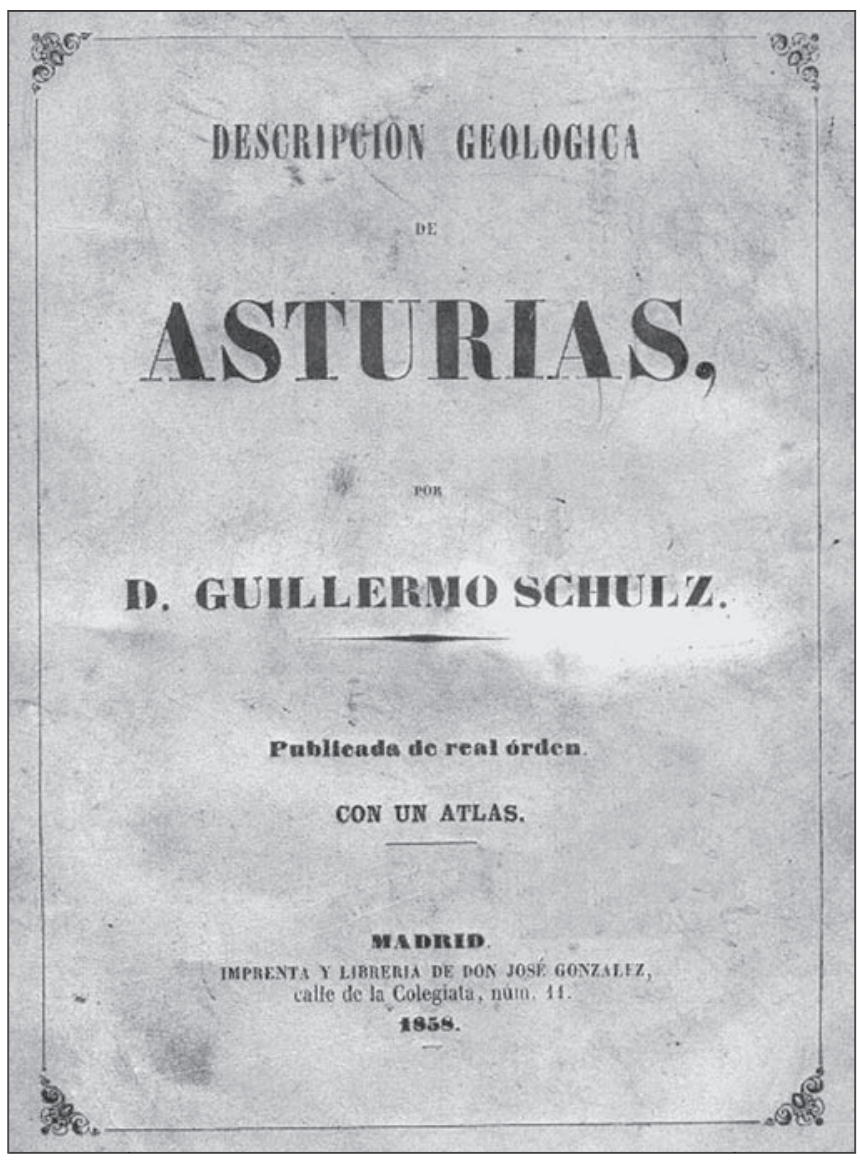

Figura 7. Portada de la Descripcion geológica de Asturias de Guillermo Schulz (1858). En esta obra se describe como perteneciente a Squalus un supuesto diente de terópodo, que podría representar el hallazgo más antiguo documentado de un fósil de dinosaurio en la Península Ibérica.

Title page of the Descripcion geológica de Asturias by Schulz (1858). In this book, a supposed theropod tooth was described as belonging to Squalus. It could be the earliest known discovery of a dinosaur fossil in the Iberian Peninsula.

paleontológica (Chure \& McIntosh, 1989). Aunque se han formalizado numerosas especies, el espécimen tipo, un dentario descubierto en el Jurásico medio de Oxfordshire (Inglaterra), descrito originalmente por Buckland (1824), está aún por revisar. Por ello, es aconsejable no asignar material adicional a este taxón hasta la publicación de una diagnosis (Allain \& Chure, 2002).

Egozcue (1873) indicó que el diente de Asturias formaba parte de las colecciones de la Escuela de Minas de Madrid. Creada en Almadén (Ciudad Real) por Carlos III, la Escuela de Minas se trasladó a Madrid en 1836. Desde esa fecha y hasta finales del siglo XIX, época en la que se construyó el edificio monumental que alberga hoy en día el Museo Histórico-Minero Don Felipe de Borbón y Grecia y la Escuela Técnica Superior de Ingenieros de Minas
(Universidad Politécnica de Madrid) en la calle Ríos Rosas (véase Calvo, 2002), las colecciones paleontológicas y mineralógicas se depositaron sucesivamente en varias sedes madrileñas. La Colección del Marqués de Elduayen, que consta de varios miles de ejemplares, la mayoría de los cuales corresponden a minerales, y en la que supuestamente se encontraría el diente de Ruedes, fue donada al Museo a través del Marqués de la Ribera hacia 1870 (B. Calvo, com. pers.). Desgraciadamente, la revisión de las colecciones de paleontología de vertebrados del Museo Histórico Minero y del Museo Geominero de Madrid ha resultado infructuosa, por lo que se desconoce el paradero del diente descrito en primera instancia por Schulz (1858) y, más tarde, por Egozcue (1873).

Por lo que respecta a su procedencia geológica, el diente de Ruedes (Asturias) se descubrió según Schulz (1858) en las «margas rubias del Lías». Como pone de relieve Suárez Vega (1974), Schulz (1858) llamó «Liásico asturiano» a todo el Jurásico, lo que hizo que, hasta 1925, se considerara el Jurásico detrítico (Malm) como Liásico. Schulz (1858) pensó que los tramos margosos-areniscosos fosilíferos de la costa asturiana entre Gijón y Ribadesella eran del Lías inferior. No obstante, autores posteriores sitúan el Jurásico detrítico de Asturias en el Malm (Suárez Vega, 1974; García-Ramos \& Gutiérrez Claverol, 1995). Las «margas rubias» de Ruedes corresponden probablemente a la alteración de las margas negras de la Formación Lastres (J.C. García-Ramos y L. Piñuela, com. pers.), que ha sido datada como Kimmeridgiense (Valenzuela et al., 1998) y, más recientemente, como Kimmeridgiense superior-Titónico inferior (Schudack \& Schudack, 2002). El diente de Ruedes sería uno de los escasos restos directos de dinosaurios descubiertos en el Jurásico superior asturiano, cuyo registro fósil está principalmente representado por icnitas (García-Ramos et al., 2002).

\section{DISCUSIÓN Y CONCLUSIONES}

A diferencia de otros paises como Inglaterra, Francia o Estados Unidos, el descubrimiento y estudio de dinosaurios en España durante el siglo XIX fue una actividad puramente anecdótica (Sanz, 1996). Las primeras citas de restos fósiles de dinosaurios descubiertos en España datan de la década de 1870 . Vilanova $(1872,1873)$ describió huesos de Iguanodon en el Cretácico de Morella (Castellón) y Utrillas (Teruel), mientras que Egozcue (1873) asignó a Megalosaurus un diente procedente del Jurásico de Asturias (Schulz, 1858).

Resulta curioso que los primeras citas de dinosaurios en España vinieran suscitadas por la actividad de dos personajes ideológicamente antagonistas como eran Vilanova y Egozcue. Su enfrentamiento es un ejemplo del contencioso existente en aquella época entre geólogos naturalistas y 
geólogos ingenieros de minas (Solé Sabaris, 1983; Gozalo, 1993). Juan Vilanova fue el primer Catedrático de Geología y Paleontología de la Facultad de Ciencias de la Universidad Central de Madrid. Fue también miembro fundador y uno de los principales impulsores de la Sociedad Española de Historia Natural (véase Gozalo, 1993; Gozalo \& Salavert, 1995; Salavert et al., 2003). Por su parte, Justo Egozcue ocupó la cátedra de Geología y Paleontología de la Escuela de Ingenieros de Minas de Madrid y, al final de su vida, fue designado Director del Mapa Geológico de España (López de Azcona, 1992). Egozcue utilizó conceptos darwinistas (aunque sin llegar a aceptar el darwinismo en todas sus tesis) y fue un representante de lo que se ha dado en llamar el «paradigma ecológico» en biología (sensu Sala Catalá, 1984; véase Sequeiros, 1991), mientras que Vilanova, convencido antidarwinista, es un ejemplo del «paradigma fisiológico» (Gozalo, 1993).

En sus trabajos, Vilanova $(1872,1873)$ mencionó la presencia de «varios restos» (al menos un hueso largo) en Morella y dos huesos largos en Utrillas, pertenecientes a Iguanodon. La colección Vilanova de dinosaurios conservada hoy en día en el Museo Nacional de Ciencias Naturales de Madrid consta de cinco restos fósiles (MNCN 609, 610, 611, 612 y 31051) procedentes del Cretácico Inferior de Morella. No existe documentación sobre su fecha de recogida pero todo apunta a que los restos se descubrieron a finales de la década de 1860. El color rojizo de los fósiles sugiere que provienen de la Formación Arcillas de Morella, de edad Aptiense inferior. Uno de los especímenes, un centro vertebral (MNCN 611), podría pertenecer a un terópodo. Otro resto vertebral, en concreto un fragmento de espina neural (MNCN 610), se atribuye provisionalmente a un saurópodo diplodocoideo. El resto del material es indeterminado, aunque dos especímenes podrían corresponder a restos de costillas. Por lo que respecta al material de Utrillas (Teruel), supuestamente perteneciente a la colección Vilanova, sólo se conserva un único resto fósil (MNCN 32653), de difícil identificación. Los «dos huesos largos» citados por Vilanova eran en realidad dos fragmentos de un mismo hueso, una tibia de terópodo, actualmente en paradero desconocido, y de la que se conserva un dibujo realizado por Royo Gómez en el primer tercio del siglo XX en el Archivo del MNCN de Madrid. La presencia del ornitópodo Iguanodon entre los restos fósiles de la colección Vilanova no puede confirmarse.

El hallazgo más antiguo documentado de un dinosaurio en España es un diente aislado procedente de Ruedes (Asturias), interpretado originalmente como perteneciente a un tiburón (Schulz, 1858). Aunque se sabe que estuvo depositado hacia 1873 en la Escuela de Minas de Madrid, su localización actual es incierta. No puede confirmarse su atribución a Megalosaurus, tal y como sugirió Egozcue (1873), pero la descripción original sugiere que el diente perteneció probablemente a un terópodo de gran tamaño.
Los primeros estudios sistemáticos de dinosaurios ibéricos no se realizaron hasta finales de 1910 y durante la década de 1920 , teniendo como protagonista al naturalista valenciano José Royo Gómez, quien recogió y describió restos fósiles de dinosaurios y otros vertebrados en las facies Weald del Levante, publicando una veintena de notas y artículos sobre sus descubrimientos (Royo Gómez, 1920, 1921, 1926, 1927, 1928; para otras referencias de Royo Gómez véanse Sanz, 1996; Alcalá \& Alcalá, 1996; RuizOmeñaca \& Pereda Suberbiola, 1999; Gasulla, 2002; Catalá, 2003; Ruiz-Omeñaca et al., 2003; Alcalá, 2004).

\section{AGRADECIMIENTOS}

Queremos expresar nuestro agradecimiento a los Drs. Begoña Sánchez Chillón (Museo Nacional de Ciencias Naturales, Madrid), Juana Molina (Archivo del Museo Nacional de Ciencias Naturales, Madrid), Benjamín Calvo y Trinidad de Torres (Museo Histórico-Minero Don Felipe de Borbón y Grecia, Madrid), Isabel Rábano (Museo GeoMinero, Madrid), Margarita Belinchón (Museo Municipal de Ciencias Naturales de Valencia) y Anna García Forner (Museo de Geología, Universidad de Valencia), así como al Dr. Luis Alcalá (Fundación Conjunto Paleontológico de Teruel), por su generosa ayuda y por la información amablemente proporcionada durante la visita de las colecciones de sus respectivas instituciones. Nuestro agradecimiento va también dirigido al Dr. Rodolfo Gozalo (Universitat de València, Burjassot) por enviarnos documentación sobre los ilustres valencianos Juan Vilanova y José Joaquín Landerer. El Dr. Jesús Catalá (Universitat de València) nos proporcionó información sobre las actividades de José Royo Gómez y las colecciones de paleontología de Valencia. Los Drs. Jaime Truyols y José Carlos García-Ramos (Universidad de Oviedo) nos ofrecieron información sobre la geología de Asturias y las actividades de Guillermo Schulz. Gracias asimismo a Laura Piñeiro y José Lires (Universidad de Oviedo) por su colaboración. Por último, el Dr. Leandro Sequeiros (Universidad de Córdoba) nos informó sobre el antagonismo ideológico entre Vilanova y Egozcue. Nuestro agradecimiento a los Drs. Rodolfo Gozalo y Lourdes Casanovas por la revisión del manuscrito y al Dr. Peter Galton por su ayuda con el inglés. Las fotografías del material de la colección Vilanova conservado en el MNCN de Madrid se reproducen con permiso de esta institución. El primer autor agradece al Ministerio de Ciencia y Tecnología la concesión de una ayuda en el marco del Programa Ramón y Cajal para la realización de un proyecto de investigación en la UPV/EHU. La investigación del segundo autor forma parte del proyecto VECOBA (VErtebrados COntinentales del BArremiense, Ministerio de Ciencia y Tecnología, ref. BTE 2001-1746). 


\section{BIBLIOGRAFIA}

Alcalá, B. \& Alcalá, L. 1996. La paleontología castellonense explorada por José Royo y Gómez. Geogaceta, 19, 177-180.

Alcalá, L. 1998. La colección de vertebrados fósiles turolenses del Museo Nacional de Ciencias Naturales (CSIC). I Jornadas sobre el Patrimonio de la provincia de Teruel, Paleontología, Rubielos de Mora, 24-26 septiembre 1999, 1 p. (sin paginar).

Alcalá, L. 2002. La colección de vertebrados fósiles de Teruel del Museo Nacional de Ciencias Naurales (CSIC). In: $E l$ Patrimonio Paleontológico de Teruel (Coords. G. Meléndez Hevia \& E. Peñalver Mollá). Instituto de Estudios Turolenses, Teruel, 339-352.

Alcalá, L. 2004. José Royo Gómez y los estudios sobre vertebrados fósiles españoles. In: Homenaje a José Royo Gómez (1895-1961) (Coords. C. Diéguez, A. Perejón \& J. Truyols). Monografies del Consell Valencià de Cultura, Valencia, 179-207.

Allain, R. 2001. Redescription de Streptospondylus altdorfensis, le dinosaure théropode de Cuvier, du Jurassique de Normandie. Geodiversitas, 23, 349-367.

Allain, R. \& Chure, D. J. 2002. Poikilopleuron bucklandii, the theropod dinosaur from the Middle Jurassic (Bathonian) of Normandy. Palaeontology, 45, 1107-1021.

Andrews, R. C. 1932. The New Conquest of Central Asia. American Museum of Natural History, New York, $678 \mathrm{pp}$.

Antunes, M. T. \& Mateus, O. 2003. Dinosaurs of Portugal. Comptes Rendus Palevol, 2, 77-95.

Bataller, J. R. 1960. Los vertebrados del Cretácico español. Notas y Comunicaciones del Instituto Geológico y Minero de España, 60, 141-164.

Brookes, R. 1763. A new and Accurate System of Natural History. Vol. 5: The Natural History of Waters, Earths, Stones, Fossils, and Minerals, with their Virtues, Properties and Medicinal Uses: To which is added the Methods in which Linnaeus has treated these subjects. J. Newberry, London, $437 \mathrm{pp}$.

Buckland, W. 1824. Notice on the Megalosaurus or great Fossil Lizard of Stonesfield. Transactions of the Geological Society of London, 2, 390-396.

Buffetaut, E. \& Le Loeuff, J. 1994. The discovery of dinosaur eggshells in nineteenth-century France. In: Dinosaur Eggs and Babies (Eds. K. Carpenter, K.F. Hirsch \& J.R. Horner). Cambridge University Press, Cambridge, New York and Melbourne, 31-34.

Buffetaut, E., Cuny, G. \& Le Loeuff, J. 1993. The discovery of French dinosaurs. Modern Geology, 18, 161-182.

Cadbury, D. 2000. The Dinosaur Hunters: A True Story of Scientific Rivalry and the Discovery of the Prehistoric World. Fourth State, London, 374 pp. [edición española: Los Cazadores de Dinosaurios, Eds. Península, Barcelona, 2002, 428 pp.].

Calderón, S. 1876. On the Fossil Vertebrata hitherto discovered in Spain. Quarterly Journal of the Geological Society of London, 33, 124-133.
Calderón, S. 1877. Enumeración de los Vertebrados fósiles de España. Anales de la Sociedad Española de Historia Natural, Memorias, 5, 413-443.

Calvo, B. 2002. El Museo Histórico Minero Don Felipe de Borbón y Grecia. Escuela Técnica Superior de Ingenieros de Minas, Universidad Politécnica de Madrid, Madrid, 117 pp.

Calvo, J. \& Salgado, L. 1996. Rebbachisaurus tessonei sp. nov. A new Sauropoda from the Albian-Cenomanian of Argentina; new evidence on the origin of the Diplodocidae. Gaia, año 1995, 11, 13-33.

Canerot, J., Cugny, J., Pardo, G., Salas, R. \& Villena, J. 1982. Ibérica Central-Maestrazgo. In: El Cretácico de España. Universidad Complutense, Madrid, 273-344.

Casanovas, M. L. 1993. Novedades en el registro fósil de dinosaurios del Levante español. Zubía, año 1992, 10, 139-151.

Catalá J. I. 2003. Between dinosaurs and turtles. José Royo Gómez (1895-1961) and the study of fossil vertebrates in contemporary Spain. In: Proceedings of the 26th INHIGEO Symposium Meeting, Geological Resources and History (Ed. M. Serrano Pinto). Centro de Estudos de História e Filosofia da Ciência e da Técnica, Universidade de Aveiro, Aveiro e Lisboa, 201-219.

Colbert, E. H. 1968. Men and Dinosaurs: The search in field and laboratory, Dutton and Co., New York, 384 pp. [Reedición: The great dinosaur hunters and their discoveries, Dovers Publications, New York, 1984, 283 pp.]

Chure, D. J. \& McIntosh, J. S. 1989. A bibliography of the Dinosauria (exclusive of Aves) 1677-1986. Museum of Western Colorado, Paleontology Series, 1, 1-226.

Currie, P. J. 1997. Theropods. In: The Complete Dinosaur (Eds. J.O. Farlow \& M.K. Brett-Surman). Indiana University Press, Bloomington and Indianapolis, 216-233.

Cuvier, G. 1808. Sur les ossemens fossiles de crocodiles, et particulièrement sur ceux des environs du Havre et de Honfleur, avec des remarques sur les squelettes de sauriens de la Thuringe. Annales du Museum National d'Histoire Naturelle de Paris, 12, 73-110.

Cuvier, G. 1824. Recherches sur les ossemens fossiles, où l'on rétablit les caractères de plusieurs animaux dont les révolutions ont détruit les espèces. Dufour et d'Ocagne, Paris, $2^{\text {ème }}$ edition, t. 5, $2^{\text {ème }}$ série, 547 pp.

Delair, J. B. \& Sarjeant, W. A. S. 1975. The earliest discoveries of dinosaurs. Isis, 66, 5-25.

Delair, J. B. \& Sarjeant, W. A. S. 2002. The earliest discoveries of dinosaurs; the records re-examined. Proceedings of the Geologists' Association, 113, 185-197.

Dong, Z.-M. 1988. Dinosaurs from China. China Ocean Press, Beijing and British Museum (Natural History), London, $114 \mathrm{pp}$.

Egozcue, J. 1873. Noticia sobre la existencia en España de restos fósiles de Megalosaurus y de Hyaena spelaea y brunnea (Sesión del 4 de junio de 1873). Anales de la Sociedad Española de Historia Natural, Actas, 2, 29-31.

Gàmez D., Paciotti P., Colombo F. \& Salas R. 2003. La Formación Arcillas de Morella (Aptiense inferior), Cadena Ibérica oriental (España): caracterización sedimentológica. Geogaceta, 34, 191-194. 
García Forner, A., Gil, B., Guillem, J., Maroto, M. A., Martínez Pérez, C., Plasencia, P., Villena, J. A \& MárquezAliaga, A. 2002. Gestión, inventario e informatización de los fósiles de Teruel en el Museo de Geología de la Universitat de València. In: El Patrimonio Paleontológico de Teruel (Coords. G. Meléndez Hevia \& E. Peñalver Mollá). Instituto de Estudios Turolenses, Teruel, 371-383.

García-Ramos, J. C. \& Gutiérrez, M. 1995. La cobertera mesozoica-terciaria. In: Geología de Asturias (Eds. C. Aramburu \& F. Bastida). Eds. Trea, Gijón, 81-94.

García-Ramos, J. C., Lires, J. \& Piñuela, L. 2002. Dinosaurios. Rutas por el Jurásico de Asturias. La Voz de Asturias, Oviedo, 204 pp.

Gasulla, J. M. 2002. Aproximación a los restos de dinosaurios de Morella del Museo Nacional de Ciencias Naturales y otros restos. In: Actes de la XL Assemblea Intercomarcal d'Estudiosos (Coords. J. Pastor, E. Querol \& C. Ripollés). Diputació de Castelló, Morella, 2, 461-476.

Gozalo, R. 1993. Biografía de Juan Vilanova y Piera. In: Homenaje a Juan Vilanova y Piera, Valencia, 25-27 de noviembre de 1993. Universitat de València, Diputación de Valencia y Sociedad Económica de Amigos del País de Valencia, Valencia, 11-83.

Gozalo, R. \& Navarro, V. 1995. Josep Joaquim Lànderer i Climent (València, 1841 - Tortosa, 1922). La recerca fora del món acadèmic: astronomia i geologia. In: Ciència $i$ Tècnica als Països Catalans: una aproximació biogràfica (Dir. J.M. Camarasa \& A. Roca Rossell). Fundació Catalana per a la Recerca, Barcelona, 1, 459-492.

Gozalo, R. \& Salavert, V. 1995. Joan Vilanova i Piera (València, 1821 - Madrid, 1883). Geòleg, paleontòleg i prehistoriador. In: Ciència i Tècnica als Països Catalans: una aproximació biogràfica (Dir. J.M. Camarasa \& A. Roca Rossell). Fundació Catalana per a la Reçerca, Barcelona, 1, 289-313.

Halstead, L. B. \& Sarjeant, W. A .S. 1993. Scrotum humanun Brookes - The earliest name for a dinosaur? Modern Geology, 18, 221-224.

Holtz, T. R. Jr. 2000. A new phylogeny of the carnivorous dinosaurs. In: Aspects of Theropod Paleobiology (Eds. B. Pérez-Moreno, T. Holtz, Jr., J.L. Sanz \& J. Moratalla). Gaia, año 1998, 15, 5-61.

Landerer, J. J. 1874. El piso tenéncico ó urgo-áptico y su fauna. Anales de la Sociedad Española de Historia Natural, Memorias, 3, 345-386.

Lapparent, A. F. de 1960. Los dos dinosaurios de Galve. Teruel, 24, 177-197.

Lhuyd, E. 1699. Eduardi Luidii. Lithophylacii Britannici ichnographia, sive, Lapidum aliorumque fossilium Britannicorum singulari figura insignium, quotquot hactenus vel ipse invenit vel ab amicis accepit, distributio classica, scrinii sui lapidarii repertorium cum locis singulorum natalibus exhibens. Additis rariorum aliquot figuris aere incisis, cum epistolis ad clarissimos viros de quibusdam circa marina fossilia et stirpes minerales praesertim notandis. Ex officina M. C., Londini, 139 pp.

Lockley, M. G. 1991. Tracking dinosaurs: A new Look at an Ancient World, Cambridge University Press, Cambridge,
252 pp. [edición española: Siguiendo las huellas de los dinosaurios, McGraw-Hill, Madrid, 1993, 307 pp.].

López de Azcona, J. M. 1984. Mineros destacados del siglo XIX. Guillermo Schulz y Schweizer (1800-1877). Boletín Geológico y Minero, 95, 184-189.

López de Azcona, J. M. (Ed.). 1992. Egozcue y Cía, Justo. In: Bibliografía Minera Iberoamericana. ITGE, Madrid, 147-150.

Madsen, J. H. 1976. Allosaurus fragilis: A revised osteology. Bulletin of the Utah Geological and Mineral Survey, 109, 1-163.

Mallada, L. 1885. Sinopsis de las especies fósiles que se han encontrado en España. Tomo II. Terreno Mesozoico (Sistemas Triásico y Jurásico). Imprenta y Fundición de Manuel Tello, Madrid, 150 pp.

Mallada, L. 1887. Sinopsis de las especies fósiles que se han encontrado en España. Tomo III. Terreno Mesozoico (Sistema Cretáceo inferior). Imprenta y Fundición de Manuel Tello, Madrid, 174 pp

Mallada, L. 1892. Catálogo general de las especies fósiles encontradas en España. Boletín de la Comisión del Mapa Geológico de España, 18, año 1891, 1-253.

Mallada, L. 1902. Explicación del Mapa Geológico de España. Tomo IV: Sistemas Permiano, Triásico, Liásico y Jurásico. Memorias de la Comisión del Mapa Geológico de España, 22, 1-514.

Mallada, L. 1904. Explicación del Mapa Geológico de España. Tomo V: Sistemas Infracretáceo y Cretáceo. Memorias de la Comisión del Mapa Geológico de España, 23, 1-549.

Mantell, G. A. 1825. Notice on the Iguanodon, a newlydiscovered fossil reptile, from the sandstone of Tilgate Forest, in Sussex. Philosophical Transactions of the Royal Society, 115, 179-186.

Mayor, A. 2000. The First Fossil Hunters: Paleontology in Greek and Roman times. Princeton University Press, Princeton, 384 pp. [edición española: El secreto de las ánforas, Random House Mondadori - Grijalbo, Barcelona, 2002, 429 pp.].

Montero, A. 2003. La Paleontología y sus colecciones desde el Real Gabinete de Historia Natural al Museo Nacional de Ciencias Naturales. Monografías del Museo Nacional de Ciencias Naturales. Consejo Superior de Investigaciones Científicas, Madrid, 19, 383 pp.

Navarro, V. \& Catalá, J. 2000. Las ciencias. In: Historia de la Universidad de Valencia. Volumen III. La universidad liberal (siglos XIX y XX) (Coord. M. Peset). Universitat de València, Valencia, 149-176.

Ortí Miralles, F. 1958. Historia de Morella. Tomo I: Prehistoria y Protohistoria. Ediciones Ortí, Benimodo (Valencia), $135 \mathrm{pp}$.

Owen, R. 1842. Report on British Fossil Reptiles: Part II. Report of the British Association for the Advancement of Science, 1841, 60-204.

Plot, R. 1677. The Natural History of Oxfordshire, being an essay towards the Natural History of England. Published by the author, printed at The Theater, Oxford, 358 pp.; $2^{\text {nd }}$ ed., Blume, London, 1705. 
Probst, E. \& Windolf, R. 1993. Dinosaurier in Deutschland. C. Bertelsmann Verlag, München, 316 pp.

Puche Riart, O \& Ayala-Carcedo, F. J. 2001. Guillermo P. D. Schulz y Schweizer (1800-1877): su vida y su obra en el bicentenario de su nacimiento. Boletín Geológico y Minero, 112, 105-122.

Querol, X., Salas, R., Pardo, G. \& Ardèvol, L. 1992. Albian coal-bearing deposits of the Iberian Range in northeastern Spain. Geological Society of America Special Paper, 267, 193-208.

Quiroga, F. 1893. El profesor D. Juan Vilanova y Piera (Sesión del 4 de octubre de 1893). Anales de la Sociedad Española de Historia Natural, Actas, 22, 132-137.

Royo Gómez, J. 1920. Los yacimientos weáldicos del Maestrazgo. Boletín de la Sociedad Española de Historia Natural, 20, 261-267.

Royo Gómez, J. 1921. Las Facies continental en el Cretácico inferior Ibérico. Asociación Española para el Progreso de las Ciencias, Congreso de Oporto, 4, 221-235.

Royo Gómez, J. 1926. Los vertebrados del Cretácico español de facies weáldica. Boletín del Instituto Geológico de España, 47, 171-176.

Royo Gómez, J. 1927. Sur le faciès wealdien d'Espagne. Compte Rendu sommaire des Séances de la Société géologique de France, 11, 125-128.

Royo Gómez, J. 1928. Les vertébrés du faciès wealdien espagnol. Comptes Rendus du XIV Congrès géologique International, Madrid, año 1926, 4, 2039-2042.

Ruiz-Omeñaca, J. I. \& Pereda Suberbiola, X. 1999. Un documento inédito de Royo y Gómez sobre los dinosaurios del Levante. Temas Geológico-Mineros, 26, 111-112.

Ruiz-Omeñaca, J. I., Pereda Suberbiola, X. \& Company, J. 2003. Juan Vilanova, José Royo y Gómez y los dinosaurios de Morella en las colecciones del Museo Nacional de Ciencias Naturales de Madrid. Libro de Resúmenes de las XIX Jornadas de Paleontología, Morella 2003, 150-151.

Ruiz-Omeñaca, J. I. \& Santos Cubedo, A. 1998. Un húmero de iguanodóntido (Ornithischia: Ornithopoda) del Cretácico inferior (Aptiense) de Morella (Castellón, España). Geogaceta, 24, 279-282.

Sala Catalá, J. 1984. Conflictos y paradigmas en la biología de la segunda mitad del siglo XIX. Actas del II Congreso de la Sociedad Española de Historia de la Ciencia, Jaca, 3, 277-291.

Salavert, V. L., Pelayo, F. \& Gozalo, R. 2003. Los inicios de la Prehistoria en la España del siglo XIX: Juan Vilanova y Piera y el origen y antigüedad del hombre. Fundación Marcelino Botín, Universitat de València. Instituto de Historia de la Ciencia y Documentación, CSIC - Universitat de València, Valencia, CD-ROM.

Santafé, J. V. \& Casanovas, M. L. 1993. Dinosaurios en la Comunidad Valenciana. Generalitat Valenciana, Valencia, 205 pp.

Santafé, J. V., Casanovas, M. L., Sanz, J. L. \& Calzada, S. 1982. Geología y Paleontología (Dinosaurios) de las Capas Rojas de Morella (Castellón, España). Diputación Provincial de Castellón y Diputació de Barcelona, Castellón y Barcelona, 169 pp.
Sanz, J. L. 1984. Las faunas españolas de dinosaurios. I Congreso Español de Geología, 1, 497-506.

Sanz, J. L. 1996. José Royo y Gómez y los dinosaurios españoles. Geogaceta, 19, 167-170.

Sanz, J. L. 1998. El estudio de los dinosaurios en España. Quercus, 148, 16-22.

Sanz, J. L., Buscalioni, A. D., Moratalla, J. J., Francés, V. \& Antón, M. 1990. Los reptiles mesozoicos del registro español. Monografías del Museo Nacional de Ciencias Naturales. Consejo Superior de Investigaciones Científicas, Madrid, 2, 1-79.

Sarjeant, W. A. S. 1997. The earliest discoveries. In: The Complete Dinosaur (Eds. J.O. Farlow \& M.K. BrettSurman). Indiana University Press, Bloomington and Indianapolis, 3-11.

Schudack, U. \& Schudack, M. 2002. New biostratigraphical data for the Upper Jurassic of Asturias (Northern Spain) based on Ostracoda. Revista Española de Micropaleontología, 34, 1-18.

Schulz, G. 1858. Descripcion geológica de Asturias (Descripción geológica de la Provincia de Oviedo). Imprenta de D. José González, Madrid, 138 pp.

Segura Barreda, J. 1868. Morella y sus aldeas: corografía, estadística, historia, tradiciones, costumbres, industria, varones ilustres etc. de esta antigua población y de las que fueron sus aldeas. Tomo 1. Imprenta de F. Javier Soto, Morella, $456 \mathrm{pp}$.

Sequeiros, L. 1991. Dos paradigmas paleontológicos en la ciencia española del siglo XIX. El enfrentamiento entre Justo Egozcue y Juan Vilanova y Piera. Actas V Congreso de la Sociedad Española de Historia y de las Ciencias y de las Técnicas, Murcia, 18-21 de diciembre de 1989, 2, 929-942.

Solé Sabaris, L. 1983. Los más antiguos mapas geológicos de España. Mundo Científico, 23, 252-262.

Suárez Vega, L. C. 1974. Estratigrafía del Jurásico de Asturias. Cuadernos de Geología Ibérica, 3, 1-369.

Taquet, P. 1984. Cuvier - Buckland - Mantell et les dinosaures. In: Actes du Symposium paléontologique Georges Cuvier (Eds. E. Buffetaut \& E. Salmon), Montbéliard, 475-491.

Taquet, P. 2001. Philippe Matheron et Paul Gervais: deux pionniers de la découverte et de l'étude des os et des oeufs de dinosaures de Provence (France). Geodiversitas, 23, 611-623.

Truyols, J. 1995. El conocimiento histórico de la geología asturiana. In: Geología de Asturias (Eds. C. Aramburu \& F. Bastida). Eds. Trea, Gijón, pp. 11-26.

Upchurch, P. 1998. The phylogenetic relationships of sauropod dinosaurs. Zoological Journal of the Linnean Society, 124, 43-103.

Valenzuela, M., Díaz González, T. E., Gutiérrez Villarias, M. I. \& Suárez de Centi, C. 1998. La Fm. Lastres del Kimmeridgiense de Asturias: Sedimentología y estudio paleobotánico inicial. Cuadernos de Geología Ibérica, 24, 141-171.

Vilanova, J. 1872. Compendio de Geología. Imprenta de Alejandro Gómez Fuentenebro, Madrid, 588 pp.

Vilanova, J. 1873. Restos de Iguanodon en Utrilla (Sesión del 
5 de febrero de 1873). Anales de la Sociedad Española de Historia Natural, Actas, $2,8$.

Vilanova, J. 1876. Tomo VIII: Mineralogía, Geología y Paleontología. In: La Creación. Historia Natural, escrita por una sociedad de Naturalistas y publicada bajo la dirección de Juan Vilanova y Piera. Montaner y Simón, Barcelona, iv + 484 pp.
Wilson, J. A. 2002. Sauropod dinosaur phylogeny: critique and cladistic analysis. Zoological Journal of the Linnean Society, 136, 217-276.

Manuscrito recibido: 19 de Febrero, 2004 Manuscrito aceptado: 19 de Mayo, 2005 